\title{
Checklist da Flora de Mirandiba, Pernambuco: Leguminosae ${ }^{1}$
}

\author{
Elisabeth Córdula ${ }^{2,4}$, Luciano Paganucci de Queiroz ${ }^{3} \&$ Marccus Alves $^{2}$
}

\section{RESUMO}

(Checklist da Flora de Mirandiba, Pernambuco: Leguminosae) A família Leguminosae é a mais representativa da caatinga, compreendendo cerca de um terço da riqueza de espécies catalogadas. Devido à importância das Leguminosae no bioma, foi realizado um inventário florístico no município de Mirandiba-PE, área considerada prioritária para investigação científica devido à carência de informação sobre a flora e a fauna. A coleta de material botânico foi realizada entre março de 2006 e janeiro de 2008. Foram registradas 81 espécies distribuídas em 42 gêneros representando cerca de $25 \%$ das leguminosas já citadas para o bioma. Destas, 17 são endêmicas da caatinga, representando $25 \%$ do total de espécies amostradas. A subfamília Caesalpinioideae contribuiu com 22 espécies distribuídas em sete gêneros, Mimosoideae com 23 espécies distribuídas em 13 gêneros e Papilionoideae com 36 espécies distribuídas em 22 gêneros. Os gêneros mais representativos foram Senna (8 spp.), Mimosa (7 spp.), Chamaecrista (6 spp.), Macroptilium (4 spp.), Aeschynomene, Caesalpinia, Centrosema, Senegalia e Zornia (3 spp. cada), Bauhinia, Chloroleucon, Crotalaria, Desmodium, Dioclea, Indigofera, Piptadenia e Stylosanthes (2 spp. cada), e os demais com uma espécie cada.

Palavras-chave: diversidade, caatinga, endemismo.

\section{Abstract}

(Checklist of the Flora of Mirandiba: Leguminosae) The Family Leguminosae is the most representative of the caatinga, comprizing about a third of the total number of species detected for that biome. Due to the importance of Leguminosae in caatinga, a floristic inventory was accomplished in the municipal district of Mirandiba-PE, a prioritary area for scientific investigation due to the lack of information on the flora and the fauna. Field trips were accomplished from March 2006 to January 2008 for collecting botanical material. Eighty one species distributed in 42 genera were registered, comprizing about $25 \%$ of the Leguminosae already registered for the biome. Seventeen of them are endemic to the caatinga, representing $25 \%$ of the total sampled species. The subfamily Caesalpinioideae is represented by 22 species distributed in 7 genera, Mimosoideae by 23 species distributed in 13 genera and Papilionoideae by 36 species distributed in 22 genera. The most representative genera were Senna (8 spp.), Mimosa (7 spp.), Chamaecrista (6 spp.), Macroptilium (4 spp.), Aeschynomene, Caesalpinia, Centrosema, Senegalia and Zornia (3 spp.), Bauhinia, Chloroleucon, Crotalaria, Desmodium, Dioclea, Indigofera, Piptadenia and Stylosanthes (2 spp.), the other ones with one species each.

Key words: diversity, caatinga, endemism.

\section{INTRODUÇÃo}

A caatinga é a vegetação predominante no Nordeste do Brasil (Prado 2003), o clima é semi-árido (BShw', segundo Köppen) com um alto potencial de evapotranspiração (1500-2000 mm/ano) e precipitação escassa (300-1000 mm/ano) normalmente concentrado de 3-5 meses (Sampaio 1995). Os solos podem ser litólicos, podzólicos vermelho-

amarelos rasos ou brunos não-cálcicos, provenientes de embasamento cristalino, ou arenoquartzosos muito profundos e fortemente drenados provenientes de bacias sedimentares (Beltrão \& Lamour 1985).

De acordo com MMA (2002), a caatinga apresenta uma heterogeneidade marcante com várias fisionomias o que faz dela um ambiente de extrema importância biológica.

Artigo recebido em 02/2008. Aceito para publicação em 05/2008.

${ }^{1}$ Parte da dissertação de mestrado da primeira autora no PPGBV - UFPE

${ }^{2}$ Laboratório de Morfo-Taxonomia Vegetal, Departamento de Botânica, UFPE, Av. Prof. Moraes Rego, s/n, 50670-901, Recife, PE, Brasil.

${ }^{3}$ Universidade Estadual de Feira de Santana, Departamento de Ciências Biológicas, km 03, BR 116, 44031-460, Feira de Santana, BA, Brasil.

${ }^{4}$ Autor para correspondência: ecordula@yahoo.com.br 
A vegetação pode ser caracterizada como uma floresta baixa composta principalmente por árvores pequenas e arbustos. Freqüentemente, os caules retorcidos, além da presença de espinhos e microfilia, sendo decíduos na estação seca. Plantas suculentas da família Cactaceae são comuns e a camada herbácea é efêmera, só estando presente durante a estação chuvosa. Ao contrário do postulado, a caatinga apresenta uma alta taxa de diversidade e endemismo, fazendo-se necessário um melhor conhecimento de sua flora para possíveis medidas de conservação de suas áreas (Prado 2003). Este é o bioma menos estudado entre as regiões fitogeográficas brasileiras e o menos protegido pelas unidades de conservação e proteção integral (Leal et al. 2003).
A família Leguminosae é de grande relevância para a caracterização fisionômica dos diversos ambientes no domínio das caatingas. Estudos recentes enfatizam a distinção da flora de Leguminosae em diferentes tipos de sedimento (Queiroz 2006; Cardoso \& Queiroz 2007), abrindo caminho para uma nova visão em termos de florística e estimulando estudos de análise de similaridade. Na caatinga, estão catalogados até o momento 77 gêneros e cerca de 300 espécies, representando aproximadamente um terço da vegetação (Queiroz 2006) e diversos representantes possuem grande potencial econômico como recurso forrageiro durante a seca, por ser o principal componente da diversidade vegetal do ambiente (Queiroz 1999).

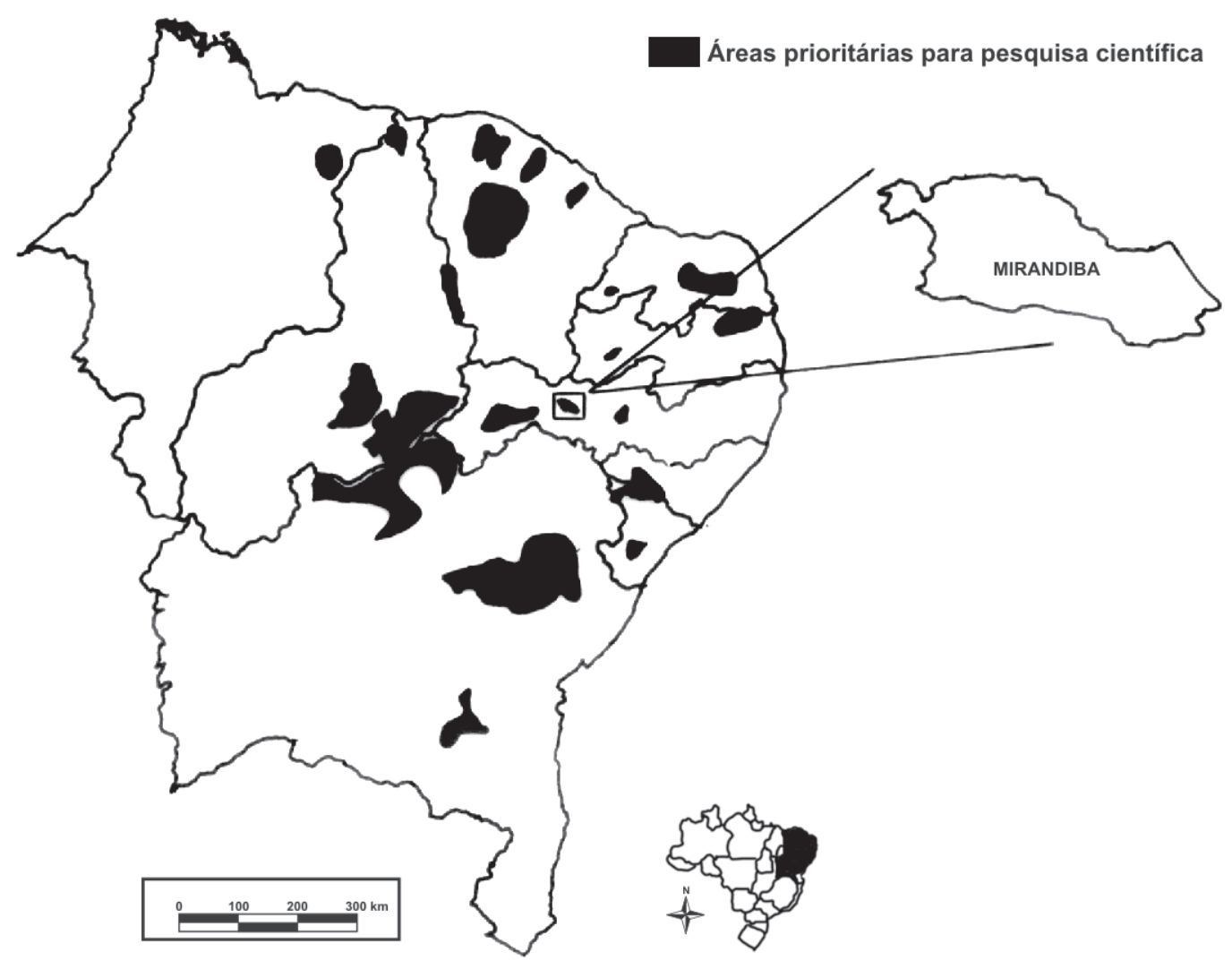

Figura 1 - Mapa da Região Nordeste do Brasil destacando as áreas prioritárias para a pesquisa cientifica e o Município de Mirandiba - PE. Fonte: MMA 2002. 
Neste sentido, realizou-se o levantamento das Leguminosae ocorrentes em Mirandiba, com o intuito de contribuir para o conhecimento da flora local.

\section{Material e Métodos}

O município de Mirandiba foi considerada pelo MMA (2002) como prioritária para investigação científica devido à carência de informação sobre a flora e a fauna. Situa-se na mesorregião do Sertão Pernambucano, próximo ao limite norte da ecorregião da Depressão Sertaneja Meridional (Fig. 1). Ocupa uma área de $809 \mathrm{~km}^{2}$, altitude média de $550 \mathrm{~m}$ e coordenada central $08^{\circ} 13^{\prime} \mathrm{S}$ e $38^{\circ} 43^{\prime} \mathrm{W}$. O clima da região é BShw' segundo Köppen, com chuvas concentradas de dezembro a março, com temperatura média anual de $25,2^{\circ} \mathrm{C}$ (ITEP).

Foram realizadas coletas de material botânico buscando percorrer a maior área possível entre março de 2006 e janeiro de 2008 , além de levantamento nos herbários de referência para a caatinga (HUEFS, IPA, JPB, PEUFR e UFP). Todas as amostras, com exceção de EH 865 (IPA) e EH 783 (PEUFR), foram tombadas ao acervo do herbário da Universidade Federal de Pernambuco, com duplicatas no HUEFS, IPA, JPB, K, NY e RB (acrônimos de acordo com Holmgren \& Holgren 1998).

Adotou-se a classificação de Leguminosae em subfamílias proposta por Lewis et al. (2005).

\section{Resultados e Discussão}

O estudo da composição florística em Mirandiba resultou em 81 espécies de Leguminosae distribuídas em 42 gêneros (Tab. 1). Isto representa cerca de $20 \%$ dos táxons citados para a caatinga e cerca de $40 \%$ das espécies indicadas para a Depressão Sertaneja Meridional, onde o Município se insere (Queiroz 2006). Das 81 espécies amostradas, 17 são endêmicas da caatinga, representando $25 \%$ do total.
Caesalpinioideae está representada em Mirandiba por 22 espécies distribuídas em sete gêneros. Senna é o mais bem representado com oito espécies, seguido de Chamaecrista (6 spp.), Caesalpinia (3 spp.), Bauhinia (2 spp.) e os demais com uma espécie cada. Apenas quatro espécies haviam sido até então coletadas na área, Chamaecrista acosmifolia, Senna macranthera, S. spectabilis e Caesalpinia bracteosa. Mimosoideae está representada em Mirandiba por 23 espécies distribuídas em 13 gêneros. Mimosa é o gênero melhor representado (7 spp.), seguido por Senegalia (3 spp.), Chloroleucon e Piptadenia (2 spp. cada). Os demais gêneros estão representados por apenas uma espécie. Não havia registros conhecidos de Mimosoideae para a área de estudo. A subfamília Papilionoideae está representada em Mirandiba por 36 espécies distribuídas em 22 gêneros. O gênero Macroptilium é o mais bem representado com quatro espécies, seguido de Aeschynomene, Centrosema e Zornia com três espécies cada, Crotalaria, Desmodium, Dioclea, Indigofera e Stylosanthes com duas espécies e os demais com uma espécie cada. Apenas três espécies haviam sido coletadas ou citadas na área anteriormente, Arachis dardani (Krapovickas \& Gregory 1994), Macroptilium martii e Tephrosia purpurea subsp. purpurea, coletadas em áreas antropizadas ao longo de estradas.

Chamaecrista pilosa var. luxurians e Desmodium procumbens constituem o primeiro registro para a caatinga, assim como Neptunia plena é o primeiro registro para a caatinga de Pernambuco. Chamaecrista acosmifolia var. acosmifolia, Chamaecrista duckeana, Senegalia riparia, Galactia striata, Indigofera microcarpa e Tephrosia purpurea subsp. purpurea são novas ocorrências para o estado de Pernambuco. Tendo em vista o grande número de espécies endêmicas da caatinga e o registro de uma espécie na lista de espécies ameaçadas de extinção (Amburana cearensis), acreditamos que esta seja uma área de extrema importância para a conservação da caatinga. 
Tabela 1 - Lista das espécies de Leguminosae de Mirandiba. (Sigla para os coletores: EC = E. Córdula et al.; $\mathrm{CL}=C$. Lourenço et al.; KP $=$ K. Pinheiro et al.; JS $=J$. Silva et al.; $\mathrm{EH}=E . P$. Heringer). * Espécies endêmicas da caatinga (Queiroz 2006; Cardoso \& Queiroz 2007).

\begin{tabular}{|c|c|c|}
\hline Táxons & Hábito & Voucher \\
\hline Aeschynomene evenia Wright var. evenia & Erva & $E C 70$ \\
\hline Aeschynomene mollicula Kunth & Erva & EC 259 \\
\hline Aeschynomene viscidula Michx. & Erva & EC 32 \\
\hline Amburana cearensis (Allemão) A.C.Sm. & Árvore & EC 198 \\
\hline Anadenanthera colubrina (Vell.) Brenan & Árvore & EC 15 \\
\hline Arachis dardani Krapovickas \& W.C. Gregory * & Erva & EH 865 \\
\hline Bauhinia acuruana Moric. & Arbusto & $E C 44$ \\
\hline Bauhinia cheilantha (Bong.) Steud. & Arbusto & EC 08 \\
\hline Caesalpinia bracteosa Tul. & Arvóre & EC 77 \\
\hline Caesalpinia ferrea Mart. ex Tul. var. ferrea $*$ & Árvore & EC 10 \\
\hline Caesalpinia gardneriana Benth. * & Árvore & EC 253 \\
\hline Calliandra depauperata Benth. * & Arbusto & EC 89 \\
\hline Canavalia brasiliensis Mart. ex Benth. & Liana & EC 59 \\
\hline Centrosema pascuorum Mart. ex Benth. & Erva & CL 264 \\
\hline Centrosema rotundifolium Mart. ex Benth. & Subarbusto & EC 362 \\
\hline Centrosema virginianum (L.) Benth. & Trepadeira & EC 69 \\
\hline Chaetocalyx scandens var. pubescens (DC.) Rudd & Trepadeira & EC 274 \\
\hline $\begin{array}{l}\text { Chamaecrista acosmifolia (Mart. ex Benth.) H.S.Irwin } \\
\text { \& Barneby var. acosmifolia }\end{array}$ & Arbusto & EH 783 \\
\hline Chamaecrista amisciella (H.S.Irwin \& Barneby) H.S.Irwin \& Barneby * & Erva & EC 250 \\
\hline Chamaecista calycioides (Coll.) Greene var. calycioides & Erva & EC 28 \\
\hline Chamaecrista duckeana (P. Bezerra \& A. Fern.) H.S.Irwin \& Barneby * & Erva & EC 233 \\
\hline Chamaecrista pilosa var. luxurians (Benth.) H.S.Irwin \& Barneby & Erva & EC 55 \\
\hline Chamaecrista rotundifolia (Pers.) Greene var. rotundifolia & Erva & EC 33 \\
\hline Chloroleucon dumosum (Benth.) G.P. Lewis & Árvore & KP 249 \\
\hline Chloroleucon foliolosum (Benth.) G.P. Lewis & Árvore & EC 187 \\
\hline Crotalaria bahiaensis Windler \& S.Skinner & Arbusto & EC 258 \\
\hline Crotalaria incana $\mathrm{L}$. & Arbusto & EC 54 \\
\hline Desmanthus pernambucanus (L.) Thell. & Arbusto & EC 56 \\
\hline Desmodium glabrum (Mill.) DC. & Arbusto & EC 292 \\
\hline Desmodium procumbens (Mill.) Hitchc. & Erva & $E C 66$ \\
\hline Dioclea grandiflora Mart. ex Benth.* & Liana & EC 23 \\
\hline Dioclea violacea Mart. ex Benth. & Liana & $E C 85$ \\
\hline Enterolobium contortisiliqüum (Vell.) Morong. & Árvore & EC 178 \\
\hline Erythrina velutina Willd. & Árvore & EC 199 \\
\hline Galactia striata (Jacq.) Urban & Trepadeira & EC 67 \\
\hline Hymenaea courbaril L. & Árvore & EC 345 \\
\hline Indigofera microcarpa Desv. & Erva & EC 231 \\
\hline Indigofera suffruticosa Mill. & Arbusto & EC 17 \\
\hline Inga vera Willd. & Árvore & EC 340 \\
\hline
\end{tabular}




\begin{tabular}{|c|c|c|}
\hline Táxons & Hábito & Voucher \\
\hline Lonchocarpus araripensis Benth.* & Árvore & EC 205 \\
\hline Luetzelburgia auriculata (Allemão) Ducke* & Árvore & EC 176 \\
\hline Macroptilium bracteatum (Nees \& Mart.) Maréchal \& Baudet & Trepadeira & EC 68 \\
\hline Macroptilium gracile (Poepp. ex Benth.) Urban & Erva & EC 227 \\
\hline Macroptilium lathyroides (L.) Urban & Erva & EC 39 \\
\hline Macroptilium martii (Benth.) Maréchal \& Baudet & Trepadeira & EC 60 \\
\hline Mimosa arenosa (Willd.) Poir. & Arbusto & EC 29 \\
\hline Mimosa invisa Mart. ex Colla. & Subarbusto & EC 83 \\
\hline Mimosa modesta var. ursinoides (Harms) Barneby & Erva & EC 360 \\
\hline Mimosa ophtalmocentra Mart. ex Benth.* & Arbusto & $J S 193$ \\
\hline Mimosa quadrivalvis var. leptocarpa (DC.) Barneby & Subarbusto & EC 57 \\
\hline Mimosa sensitiva $\mathrm{L}$. & Subarbusto & EC 266 \\
\hline Mimosa tenuiflora (Willd.) Poir. & Arbusto & EC 87 \\
\hline Neptunia plena (L.) Benth. & Arbusto & EC 288 \\
\hline Parapiptadenia zehntneri (Harms) M.P.Lima \& H.C.Lima* & Árvore & EC 203 \\
\hline Parkinsonia aculeata $\mathrm{L}$. & Arbusto & EC 90 \\
\hline Piptadenia stipulacea (Benth.) Ducke* & Arbusto & EC 257 \\
\hline Piptadenia viridiflora (Kunth.) Benth. & Arbusto & EC 330 \\
\hline Pithecellobium diversifolium Benth.* & Árvore & EC 196 \\
\hline Pityrocarpa moniliformis (Benth.) Luckow \& Jobson & Árvore & EC 24 \\
\hline Poeppigia procera Presl. & Árvore & EC 25 \\
\hline Rhynchosia minima (L.) DC. & Trepadeira & EC 36 \\
\hline Senegalia piauhiensis (Benth.) A.Bocage \& L.P.Queiroz* & Arbusto & EC 43 \\
\hline Senegalia polyphylla (DC) Britton \& Rose & Árvore & EC 355 \\
\hline Senegalia riparia (Kunth.) Britton \& Rose ex Britton \& Killip & Arbusto & EC 190 \\
\hline Senna alata (L.) Roxb. & Arbusto & EC 65 \\
\hline Senna macranthera var. pudibunda (Benth.) H.S.Irwin \& Barneby* & Arbusto & EC 307 \\
\hline Senna obtusifolia (L.) H.S.Irwin \& Barneby & Erva & EC 222 \\
\hline Senna occidentalis (L.) Link & Arbusto & EC 280 \\
\hline Senna spectabilis var. excelsa (Schrad.) H. S. Irwin \& Barneby & Árvore & EC 46 \\
\hline Senna splendida var. gloriosa H.S.Irwin \& Barneby & Arbusto & EC 270 \\
\hline Senna trachypus (Benth.) H.S.Irwin \& Barneby * & Arbusto & EC 81 \\
\hline Senna uniflora (Mill.) H.S.Irwin \& Barneby & Erva & EC 05 \\
\hline Sesbania exasperata Kunth & Arbusto & EC 291 \\
\hline Stylosantes scabra Vogel & Erva & EC 246 \\
\hline Stylosanthes viscosa Swartz & Erva & EC 47 \\
\hline Tephrosia purpurea (L.) Pers. subsp. purpurea & Arbusto & EC 37 \\
\hline Trischidium molle (Benth.) H.E.Ireland * & Arbusto & $E C 48$ \\
\hline Vigna peduncularis (Kunth.) Fawc. \& Rendle & Trepadeira & EC 309 \\
\hline Zornia brasiliensis Vogel & Erva & EC 304 \\
\hline Zornia myriadena Benth. & Erva & EC 247 \\
\hline Zornia sericea Moric. & Erva & EC 31 \\
\hline
\end{tabular}




\section{Agradecimentos}

Os autores agradecem a equipe do Laboratório de Morfotaxonomia Vegetal da UFPE pela ajuda nas coletas, a CAPES pela bolsa de Mestrado concedida à primeira autora e ao PPGBV/UFPE e à Fundação O Boticário de Proteção à Natureza pelo financiamento das viagens de campo.

\section{REFERÊNCIAS BIBLIOGRÁFICAS}

Beltrão, V. A. \& Lamour, C. 1985. Uso atual e potencial dos solos do Nordeste. Projeto Nordeste 6, SUDENE. Recife.

Cardoso, D. B. O. S. \& Queiroz, L. P. 2007. Diversidade de Leguminosae nas caatingas de Tucano, BA: implicações para a fitogeografia do semi-árido do Nordeste do Brasil. Rodriguésia 58(2): 379-371.

ITEP - Instituto de Tecnologia de Pernambuco [On line]. Homepage: http://www.itep.br/ LAMEPE.asp > Acessado em: 23/04/2006.

Krapovickas, A. \& Gregory, W. C. 1994. Taxonomia del género Arachis (Leguminosae). Bonplandia 8: 1-186.

Leal, I. R.; Tabarelli, M. \& Silva, J. M. C. 2003. Ecologia e conservação da caatinga. Ed. Universitária da UFPE, Recife, 804p.

Lewis, G. P., Schrire, B.; Mackinder, B. \& Lock, M. 2005. Legumes of the world. Royal Botanic Gardens, Kew.
MMA. 2002. Avaliação e ações prioritárias para a conservação da biodiversidade da caatinga. UFPE/Fade/Conservation Internacional do Brasil/Fundação Biodiversitas, Brasília.

Prado, D. E. 2003. As caatingas da América do Sul. In: Leal, I. R.; Tabarelli, M. \& Silva, J. M. C. (eds.). Ecologia e conservação da caatinga. Ed. Universitária da UFPE, Recife. Pp. 3-73.

Queiroz, L. P. 1999. Leguminosas de caatinga, espécies com potencial forrageiro. In: Araújo, F. D.; Prendergast, H. D. V. \& Mayo, S. J. (eds.). Plantas do Nordeste. Anais do I Workshop Geral. Royal Botanic Gardens, Kew.

Queiroz, L. P. 2006. The Brazilian caatinga: Phytogeographical patterns inferred from distribution data of the Leguminosae. In: Pennington, R. T.; Lewis, G. P. \& Ratter, J. A. (eds.). Neotropical caatingas and dry forests: Plant diversity, biogeography, and conservation. Taylor \& Francis Crc-Press, Boca Raton.

Sampaio, E. V. S. B. 1995. Overview of the brazilian caatinga. In: Bullock, S. H.; Mooney, H. A. \& Medina, E. (eds.). Seasonally dry tropical forests. Cambridge University Press, Cambridge. 